\title{
RECURRENCE PLOT STRUCTURES REFLECT MOTOR-RELATED EEG PATTERN
}

\author{
Elena Pitsik, Nikita Frolov \\ Neuroscience and Cognitive Technology Laboratory \\ Center for Technologies in Robotics and Mechatronics Components \\ Innopolis University \\ Russia \\ e.pitsik@innopolis.ru,n.frolov@innopolis.ru
}

Article history:

Received 23.10.2019, Accepted 06.12.2019

\begin{abstract}
Detection and classification of motor-related brain patterns from non-invasive electroencephalograms (EEGs) is challenging due to their non-stationarity and low signal-to-noise ratio and requires using advanced mathematical approaches. Traditionally applied methods such as time-frequency analysis and spatial filtering allow to quantify the main attribute of the motor-related brain activity - contralateral desynchronization of $\mu$-band oscillations $(8-13 \mathrm{~Hz})$ in sensorimotor cortex - by measuring EEG signal's amplitude, power spectral density, location etc. However, these features suffer from strong inter- and intra-subject variability. So, special attention is paid to the finding of stable features. In present paper, we investigate application of the recurrence plots - robust mathematical tool for nonstationary data analysis - to explore properties of motor-related EEG samples. Our goal is to show that recurrence plots are sensitive to the changes in brain activity accessed from noninvasive EEG recordings and may provide us a new context for interpretation of motor-related pattern in EEG.
\end{abstract}

\section{Key words}

Recurrence plot, recurrence quantification analysis, EEG, motor-related pattern, event-related desynchronization

\section{Introduction}

Detecting motor-related events using EEG signal is highly demanded in the area of brain-computer interfaces development for post-stroke rehabilitation and control of external devices. The progress made in this area allowed to connect motor action with certain phenomena occurring in bran activity, forming the idea of how motor task execution is reflected in human brain. The well-known attribute of the motor-related activity confirmed by the majority of studies is event-related desynchronization (ERD), which is manifested as a suppression of $\mu$-band oscillations in sensorimotor area [Neuper et al., 2006; Pfurtscheller and Neuper, 1997]. Traditionally, ERD detection is done via time-frequency analysis [Wang et al., 2004; Ince et al., 2007; Maksimenko et al., 2018b] with the decrease of spectral power density as a classification criteria [Carrera-Leon et al., 2012; Xu and Song, 2008]. Besides, various methods were applied for this purpose including spatial filtering [Wang et al., 2006], detrended fluctuation analysis [Pavlov et al., 2018; Pavlov et al., 2019], clasterization methods [Chholak et al., 2019], and artificial intelligence [Sakhavi et al., 2015; Grubov et al., 2017; Maksimenko et al., 2018a].

However, there are inherent limitations of the mentioned approaches, such as the lack of inter- and intrasubjects robustness and the computational demands. For this reason, we introduce the approach which uses concept of recurrences to characterize complexity of the process via its signal analysis. In particular, we use recurrence plots (RPs), which is an efficient tool for visualization of repeating regimes in time series [Marwan et al., 2007]. Quantification of RP allows to interpret various dynamical regimes emerging in time series in terms of signal complexity and reveal new features of these time series. In particular, RPs were applied to biological data analysis [Hirata et al., 2016; Acharya et al., 2011; Acharya et al., 2013], seismic activity analysis [Lin et al., 2015; Chelidze and Matcharashvili, 2015], chemistry [Alves et al., 2017; Facchini et al., 2009], and were especially demanded in climate research [Deng et al., 2017; Feng and Dijkstra, 2017; García-Olivares and Herrero, 2013; Panagoulia and Vlahogianni, 2018]. 
In the current study, we apply RPs to the EEG time series associated with hands movements. In our opinion, lowering amplitude of EEG $\mu$-band oscillations during motor action is also associated with less random behavior of corresponding EEG signal. In its turn, reduce of randomness in time series generates more adjacent trajectories in the phase space, which means the increase of recurrence rate. We show that measures of recurrence quantification analysis (RQA) are sensitive to transitions from random uncorrelated background EEG to motor task accomplishment, allowing to detect movement onset.

\section{Methods}

\subsection{Experimental Dataset}

In our research we used data obtained during the experiment on recording of EEG and EMG signals while performing actual movements with right and left hands. For this research we selected 10 participants using following criteria: aged 18-33, healthy, right-handed and not experienced in participating in similar experiments.

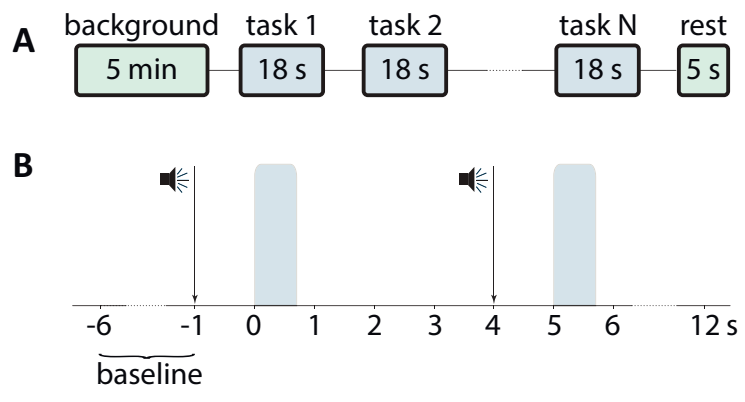

Figure 1. A - diagram of experimental design; $\mathbf{B}-$ the approximate timeline of single trial.

During the experimental session, participants were sitting in a comfortable chair with both hands lying on armrests or on the table in the pose that allows to avoid any not task-related muscle tension. Experimental session started with 5 minutes of background activity recording, which corresponds to relaxing state of participant while listening to the classical music, without focusing on any particular thought. Then, the active stage started, which consisted of two type of tasks:

1. Squeezing the left hand into fist after first short signal, holding it squeezed until the second signal, and relax it after;

2. Squeezing the right hand into fist after first long signal, holding it squeezed until the second signal, and relax it after;

Tasks were alternating randomly to avoid possible effects of adaptation, as well as distances between first and second signals within one task (4-5 seconds) and between the second signal of previous task and the first signal of next task (6-8 seconds). Finally, the experimental session ended with short period of rest. The scheme of the experimental procedure is illustrated on Fig. 1A.

All recorded signals were amplified using non-invasive EEG amplifier "Encephalan-EEGR-19/26" (Medicom MTD company, Taganrog, Russia) with sampling frequency $250 \mathrm{~Hz}$. Besides, we applied $50 \mathrm{~Hz}$ Notch filter to both EEG and EMG.

During the experimental procedure, we recorded EEG signal using $9 \mathrm{Ag} / \mathrm{AgCl}$ electrodes located on the sensorimotor cortex according to the international "10-10" system of electrode application. According to recent studies [Pfurtscheller et al., 2006] in present research we used $\mathrm{C} 3$ and $\mathrm{C} 4$ sensors as the most informative and reflecting contralateral properties of motor-related brain activity.

Before proceeding with RQA, we carried out a number of following preprocessing procedures. First, EMG from both hands were band-pass filtered in $10-100 \mathrm{~Hz}$ to capture high-frequency component of the signal associated with experimental task execution. EMG recording was necessary to capture exact moments of movement accomplishment. For further analysis, we used filtered EEG signal in the range $8-13 \mathrm{~Hz}$ to extract $\mu$-rhythm component. Whole experimental recording lasting approximately 20 minutes was sliced on trials, each $18 \mathrm{sec}$ onds long, containing 6 seconds of baseline (background activity preceding the signal), and 12 seconds fully covering the motor task. The example of single trial timing can be seen on Fig. 1B.

\subsection{Recurrence Plots}

Recurrence plot (RP) is a powerful tool for visualization and numerical analysis of repeating states emerging in time series. State $\vec{x}_{i}$ is considered as repeating, or recurrent, if it lies in some $\epsilon$-neighborhood of $\vec{x}_{j}$ in the reconstructed phase space. In other words, RPs are determining as follows:

$$
R_{i, j}(\epsilon)= \begin{cases}1, & \left\|\vec{x}_{i}-\vec{x}_{j}\right\| \leqslant \epsilon \\ 0, & \left\|\vec{x}_{i}-\vec{x}_{j}\right\|>\epsilon\end{cases}
$$

where $i, j=1, \ldots, N$ and $\epsilon$ is a recurrence threshold determining the size of considering neighborhood.

Elements of RP form various structures, each representing certain dynamical regime in time series. For quantitative analysis of these structures, Eckmann et al. [Eckmann et al., 1995] introduced recurrence quantification analysis and its measures of complexity. In present study, we use recurrence rate:

$$
R R(\epsilon)=\frac{1}{N^{2}} \sum_{i, j=1}^{N} R_{i, j}(\epsilon)
$$



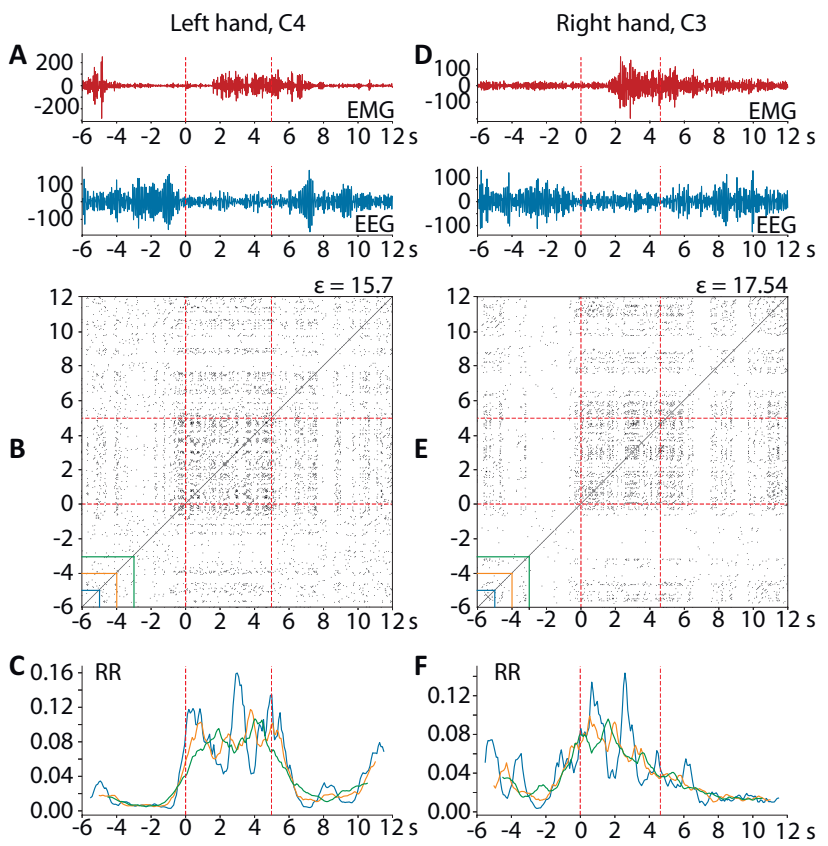

-1 second -2 seconds -3 seconds

Figure 2. A, D - raw EMG and EEG time series corresponding to the left hand (channel C4), and right hand (channel C3), respectively; $\mathbf{B}, \mathbf{E}$ - recurrence plots of corresponding time series above; $\mathbf{C}, \mathbf{F}-$ recurrence rate calculated for corresponding recurrence plots windows of different sizes. For illustrative purposes, we also show sized of used windows in lower left corner of the recurrence plots. Red dashed lines mark first and second audio command.

which measures the density of recurrence points in RP of motor-related EEG.

In our research, we compute recurrence rate in sliding window to monitor its evolution in time. Selection of window size is discussed in section 3 .

Finally, in order to validate our results we applied cluster-based nonparametric statistical test with random partitions [Maris and Oostenveld, 2007] to reveal differences between two experimental conditions (motor execution vs baseline).

\section{Results}

At the first step we demonstrate RPs of single trial EEG for right and left hands in one subject (see Fig. 2). For this illustration we selected trials with most pronounced ERD observed on raw EEG signal associated with motor execution (see Fig. 2A,D). One can notice that amplitude suppression of $\mu$-band oscillations starts even before the movement onset according to EMG, capturing short period of motor preparation after the first signal.

On Fig. 2B,E we show recurrence plots for corresponding time series (single trial for left and right hand, channels C4 and C3 respectively). Visual inspection of RPs showed increase of recurrence points density in the time interval corresponding to the motor tasks, which is consistent with our suggestion that lower signal amplitude during motor-related ERD results in larger number of neighboring trajectories in the phase space.

Since we are interested in detection of transitions from baseline to motor-related event, we use windowed modification of recurrence rate with 3 -second-window. Selection of the window size is explained on Fig. 3C,F, which presents recurrence rate calculated for windows of different sizes ( 1,2 and 3 seconds corresponding to 250,500 and 750 points of the signal). Although the measures have similar shapes, the window expansion results in more smooth form of RR, reducing uninformative fluctuations.
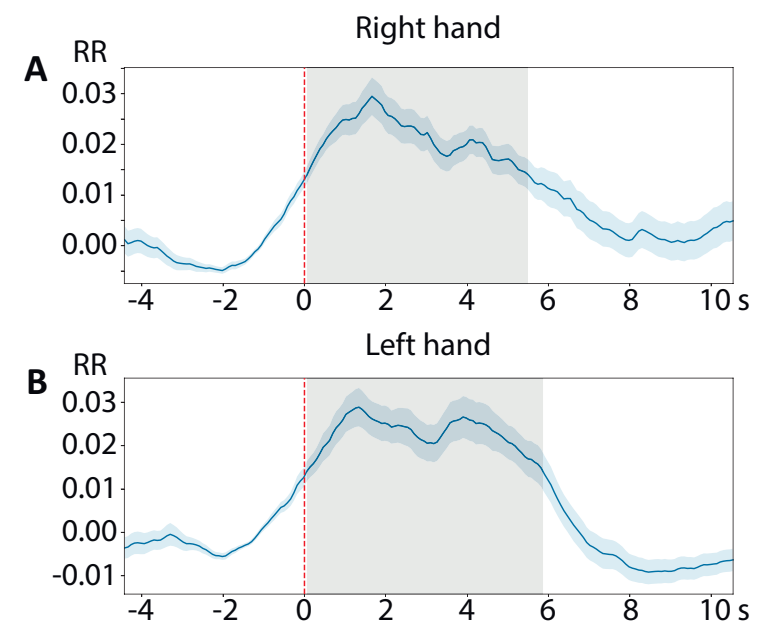

Figure 3. Recurrence rate averaged over the trials for $\mathbf{A}-$ right hand, channel $\mathrm{C} 3$ and $\mathbf{B}$ - left hand, channel $\mathrm{C} 4$ with standard error. Shaded area corresponds to the interval with statistical significant differences between baseline and motor-related EEG. Red dashed marks first audio signal.

We see that for all three window choices the recurrence rate behaves as expected, having sharp increase shortly before the signal and drop after the movement is completed. To validate this result, we perform the same analysis for recurrence rate in 3-seconds-window for whole dataset. Fig. 3 demonstrates the mean value of obtained measure with standard error and results of statistical test represented as shaded areas.

Shape of RR averaged over subjects evidences of validity of pattern observed in single trial. Increase of RR starts approximately 2 seconds before the signal, which can be explained by the shift in RR values provided by sliding window. However, area of statistically significant differences between motor accomplishment and baseline is strongly associated with motor task, since it starts almost simultaneously with the signal and ends after 5th second, when motor task is completed. Therefore, significant increase of RR is strongly associated with movement execution and even captures short period of motor preparation. We conclude, that RR not only allows 
to reveal hidden transitions associated with signal structure alternation during motor action, but also is sensitive enough to detect the movement onset using $\mu$-rhythm component of EEG signal.

\section{Conclusion}

In present paper, we demonstrated the ability of recurrence quantification analysis to detect motor-related pattern. We observed strong motor-related increase of recurrence rate peaking during the movement onset. Area of statistical significant difference between baseline and the time interval corresponding to the movement fits between two audio signals determining start and end of motor task. We believe that results of this research will be useful for further investigations of RPs use in EEG analysis, and especially in brain-computer interface applications.

\section{Acknowledgements}

This work has been supported by the Center for Technology in Robotics and Mechatronics Components (Innopolis University, Russia).

\section{References}

Acharya, U. R., Chua, E. C.-P., Faust, O., Lim, T.-C., and Lim, L. F. B. (2011). Automated detection of sleep apnea from electrocardiogram signals using nonlinear parameters. Physiological measurement, 32 (3), pp. 287.

Acharya, U. R., Faust, O., Sree, S. V., Ghista, D. N., Dua, S., Joseph, P., Ahamed, V. T., Janarthanan, N., and Tamura, T. (2013). An integrated diabetic index using heart rate variability signal features for diagnosis of diabetes. Computer methods in biomechanics and biomedical engineering, 16 (2), pp. 222-234.

Alves, L. M., Cotta, R. A., Prado, A. R., and Ciarelli, P. M. (2017). Identification of corrosive substances through electrochemical noise using wavelet and recurrence quantification analysis. In ICPRAM, pp. 718 723.

Carrera-Leon, O., Ramirez, J. M., Alarcon-Aquino, V., Baker, M., D'Croz-Baron, D., and Gomez-Gil, P. (2012). A motor imagery bci experiment using wavelet analysis and spatial patterns feature extraction. In 2012 Workshop on Engineering Applications, IEEE, pp. 16.

Chelidze, T. and Matcharashvili, T. (2015). Dynamical patterns in seismology. In Recurrence Quantification Analysis, pp. 291-334. Springer.

Chholak, P., Niso, G., Maksimenko, V. A., Kurkin, S. A., Frolov, N. S., Pitsik, E. N., Hramov, A. E., and Pisarchik, A. N. (2019). Visual and kinesthetic modes affect motor imagery classification in untrained subjects. Scientific reports, 9 (1), pp. 9838.

Deng, L., Zhang, X., An, J., and Cai, Y. (2017). Statistical properties of solar h $\alpha$ flare activity. Journal of Space Weather and Space Climate, 7, pp. A34.
Eckmann, J., Kamphorst, S. O., Ruelle, D., et al. (1995). Recurrence plots of dynamical systems. World Scientific Series on Nonlinear Science Series A, 16, pp. 441446.

Facchini, A., Rossi, F., and Mocenni, C. (2009). Spatial recurrence strategies reveal different routes to turing pattern formation in chemical systems. Physics Letters A, 373 (46), pp. 4266-4272.

Feng, Q. Y. and Dijkstra, H. A. (2017). Climate network stability measures of el niño variability. Chaos: An Interdisciplinary Journal of Nonlinear Science, 27 (3), pp. 035801.

García-Olivares, A. and Herrero, C. (2013). Simulation of glacial-interglacial cycles by simple relaxation models: consistency with observational results. Climate dynamics, 41 (5-6), pp. 1307-1331.

Grubov, V., Musatov, V. Y., Maksimenko, V., Pisarchik, A., Runnova, A., and Hramov, A. (2017). Development of intelligent system for classification of multiple human brain states corresponding to different real and imaginary movements. Cybernetics and Physics, 6, pp. 103-107.

Hirata, Y., Oda, A., Ohta, K., and Aihara, K. (2016). Three-dimensional reconstruction of single-cell chromosome structure using recurrence plots. Scientific reports, 6, pp. 34982.

Ince, N. F., Tewfik, A. H., and Arica, S. (2007). Extraction subject-specific motor imagery time-frequency patterns for single trial eeg classification. Computers in biology and medicine, 37 (4), pp. 499-508.

Lin, M., Zhao, G., and Wang, G. (2015). Recurrence quantification analysis for detecting dynamical changes in earthquake magnitude time series. International Journal of Modern Physics C, 26(07), pp. 1550077.

Maksimenko, V. A., Kurkin, S. A., Pitsik, E. N., Musatov, V. Y., Runnova, A. E., Efremova, T. Y., Hramov, A. E., and Pisarchik, A. N. (2018a). Artificial neural network classification of motor-related eeg: An increase in classification accuracy by reducing signal complexity. Complexity, 2018.

Maksimenko, V. A., Pavlov, A., Runnova, A. E., Nedaivozov, V., Grubov, V., Koronovslii, A., Pchelintseva, S. V., Pitsik, E., Pisarchik, A. N., and Hramov, A. E. (2018b). Nonlinear analysis of brain activity, associated with motor action and motor imaginary in untrained subjects. Nonlinear Dynamics, 91 (4), pp. 2803-2817.

Maris, E. and Oostenveld, R. (2007). Nonparametric statistical testing of eeg-and meg-data. Journal of neuroscience methods, 164 (1), pp. 177-190.

Marwan, N., Romano, M. C., Thiel, M., and Kurths, J. (2007). Recurrence plots for the analysis of complex systems. Physics reports, 438 (5-6), pp. 237-329.

Neuper, C., Wörtz, M., and Pfurtscheller, G. (2006). Erd/ers patterns reflecting sensorimotor activation and deactivation. Progress in brain research, 159, pp. 211222. 
Panagoulia, D. and Vlahogianni, E. I. (2018). Recurrence quantification analysis of extremes of maximum and minimum temperature patterns for different climate scenarios in the mesochora catchment in centralwestern greece. Atmospheric research, 205, pp. 33-47.

Pavlov, A., Grishina, D., Runnova, A., Maksimenko, V., Pavlova, O., Shchukovsky, N., Hramov, A., and Kurths, J. (2019). Recognition of electroencephalographic patterns related to human movements or mental intentions with multiresolution analysis. Chaos, Solitons \& Fractals, 126, pp. 230-235.

Pavlov, A., Runnova, A., Maksimenko, V., Pavlova, O., Grishina, D., and Hramov, A. (2018). Detrended fluctuation analysis of eeg patterns associated with real and imaginary arm movements. Physica A: Statistical Mechanics and its Applications, 509, pp. 777-782.

Pfurtscheller, G., Brunner, C., Schlögl, A., and Da Silva, F. L. (2006). Mu rhythm (de) synchronization and eeg single-trial classification of different motor imagery tasks. NeuroImage, 31 (1), pp. 153-159.
Pfurtscheller, G. and Neuper, C. (1997). Motor imagery activates primary sensorimotor area in humans. Neuroscience letters, 239 (2-3), pp. 65-68.

Sakhavi, S., Guan, C., and Yan, S. (2015). Parallel convolutional-linear neural network for motor imagery classification. In 2015 23rd European Signal Processing Conference (EUSIPCO), IEEE, pp. 2736-2740.

Wang, T., Deng, J., and He, B. (2004). Classifying eeg-based motor imagery tasks by means of timefrequency synthesized spatial patterns. Clinical Neurophysiology, 115 (12), pp. 2744-2753.

Wang, Y., Gao, S., and Gao, X. (2006). Common spatial pattern method for channel selelction in motor imagery based brain-computer interface. In 2005 IEEE Engineering in Medicine and Biology 27th Annual Conference, IEEE, pp. 5392-5395.

Xu, B. and Song, A. (2008). Pattern recognition of motor imagery eeg using wavelet transform. Journal of Biomedical Science and Engineering, 1 (01), pp. 64. 